\title{
Intraosseous hibernoma or unusual location of brown fat?
}

\author{
David T. Lynch • Raetasha S. Dabney • \\ Jared M. Andrews
}

Received: 11 June 2012 / Accepted: 22 August 2012 / Published online: 5 September 2012

(C) Springer-Verlag 2012

\begin{abstract}
The hibernoma is a rare benign tumor of brown fat. It is most commonly found subcutaneously in the thigh, shoulder, and back of middle-aged adults. Intraosseous hibernomas and brown fat are rarely reported. There are currently two reported cases in the literature, one of which occurred in the setting of essential thrombocythemia (ET). We report an intraosseous brown fat infiltrate found incidentally in a bone marrow biopsy of a 77-year-old female with ET.
\end{abstract}

Keywords Brown adipose tissue $\cdot$ Hibernoma $\cdot$ Intraosseous hibernoma

\section{Introduction}

The main function of brown adipose tissue is to produce heat. While once thought to only exist in infants, it has been shown to exist in normal adults as well, primarily in the neck and supraclavicular area [1]. It is also seen in a variety of other central locations in the body, such as the scapular region, mediastinum, and retroperitoneum.

Hibernomas are rare benign tumors arising from brown fat. Originally described by Merkel in 1906, the name hibernoma was coined by Gery nearly a decade later because of its resemblance to so-called hibernating glands of

D. T. Lynch $(\bowtie) \cdot$ J. M. Andrews

Department of Pathology,

San Antonio Military Medical Center,

3551 Roger Brooke Dr,

San Antonio, TX 78159, USA

e-mail: david.lynch.23@us.af.mil

\section{R. S. Dabney}

Department of Hematology and Oncology,

San Antonio Military Medical Center,

3551 Roger Brooke Dr,

San Antonio, TX 78159, USA animals. This tumor is most commonly reported in adults in the third and fourth decade, with nearly equal distributions between the sexes. They are commonly reported outside of the normal anatomic distribution of brown fat. In fact, the most common location is the thigh, followed by the shoulder, back, and neck [2]. Grossly, they appear as yellow to brown, lobulated, well-circumscribed masses often several centimeters in size.

Microscopically, hibernomas are composed of oval or polygonal cells with numerous cytoplasmic vacuoles which compress the small, centrally located nucleus. The cells may have a granular appearance when the vacuoles are small. Typically, there is a smaller population of admixed univacuolated adipocytes [3]. The largest study to date noted variations of hibernomas including spindle cell, lipomalike, and myxoid variants. A population of univacuolated cells was present in most cases. In all morphologic groups, the majority of cases showed either focal or diffuse staining for S100 [2].

Rearrangements of 11q13 can be seen in hibernomas, which is also seen in typical lipomas and myxoid liposarcoma $[4,5]$. There is no known syndrome associated with hibernomas.

Intraosseous brown fat is rarely reported; only two cases are currently in the literature. We present a case of an incidentally discovered intraosseous brown fat infiltrate of the ileum.

\section{Clinical history}

The patient is a 77-year-old female who underwent a bone marrow biopsy to evaluate a platelet count of $670,000 /$ $\mathrm{mm}^{3}$. Peripheral blood was positive for the JAK2 V617F mutation, and the patient was diagnosed with essential thrombocythemia. No clinical symptoms developed over a follow-up of 6 months. 


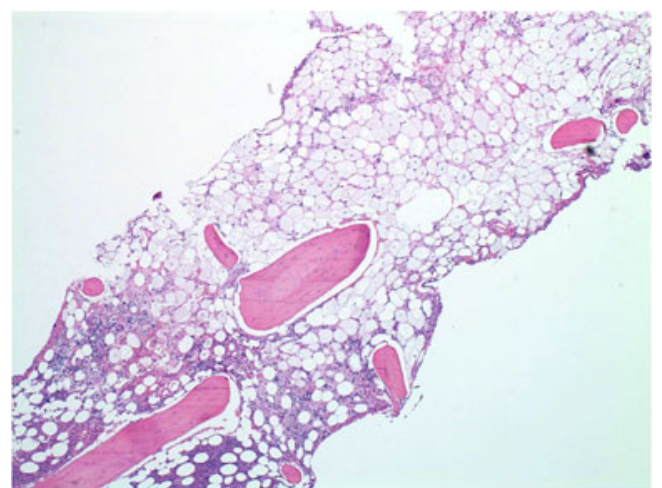

Fig. 1 Low power view of bone marrow biopsy showing replacement of hematopoietic elements $(\times 40)$

\section{Materials and methods}

A trephine biopsy of the bone marrow was taken from the left iliac crest. The core biopsy was stained with hematoxylin and eosin. Antibodies to CD68 (790-2931), p53 (790-2912), and S100 (760-2523) from Ventana (Arizona, USA) and CD31 (760-4246) from Cell Marque (California, USA) were used on formalin-fixed, paraffin-embedded tissue. Detection of S100 was performed with Ventana ultraView Universal Alkaline Phosphatase Red Detection Kit (760-501); all other antibodies were visualized with Ventana ultraView DAB detection kit (769-500). JAK2 V617F mutation analysis was performed with PCR-based quantitative pyrosequencing.

\section{Results}

The marrow core was roughly half filled with hematopoietic elements and half filled with adipose tissue (Fig. 1). The proliferation measured $0.5 \mathrm{~cm}$ and continued to the edge of the biopsy. There was circumscription of the adipose, although no capsule was noted. Closer inspection revealed a proliferation of adipocytes with numerous small vacuoles and nuclei that were small, centrally placed, and indented

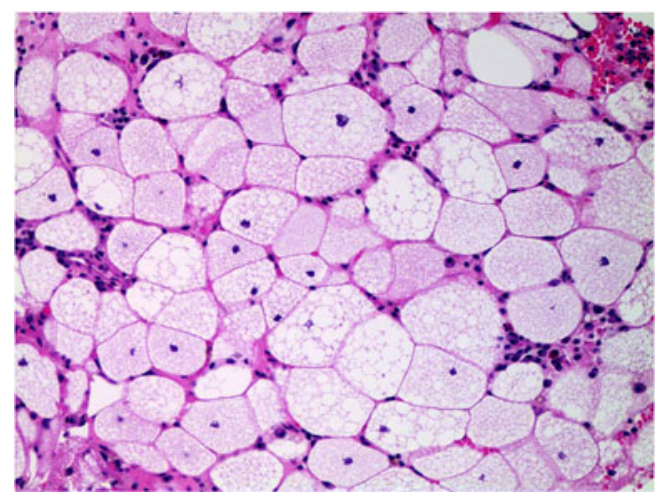

Fig. 2 High power view of the multivacuolated cells with central nuclei with routine hematoxylin and eosin stain $(\times 200)$

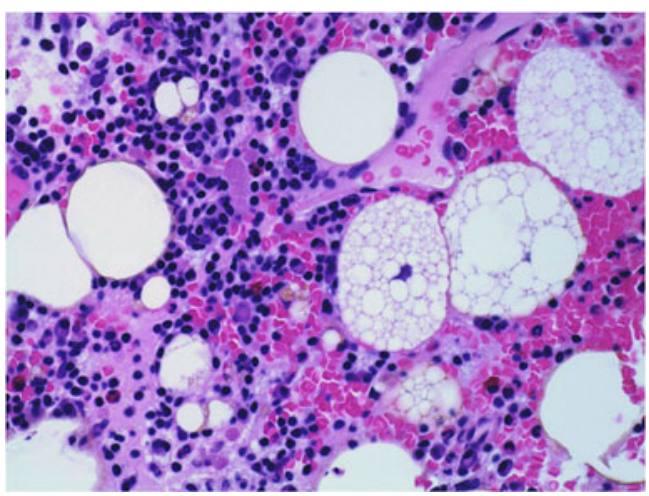

Fig. 3 High power view showing the interface of the brown fat cells with hematopoietic elements $(\times 400)$

(Figs. 2 and 3). Univacuolated cells composed 10 to $20 \%$ of the area. Scattered plasma cells were present. An S100 immunohistochemical stain showed diffuse positivity (Fig. 4). Staining for CD68, CD31, and p53 were negative.

\section{Discussion}

Hibernomas are typically subcutaneous or intramuscular. Only one prior exception, to our knowledge, was intraosseous. This case was in the sacrum of a 57-year-old female who presented with low back pain. It was evaluated with an MRI which showed a round 2-cm lesion. Her symptoms improved with analgesics [6]. It is not reported if she was diagnosed with any myeloproliferative disorder. A single case of hibernoma-like brown fat within the marrow space is reported upon an incidental finding in a 61-year-old female whose bone marrow biopsy was performed for the diagnosis of ET. The JAK2 V167F mutation was present [7].

Both of these cases showed S100 positivity. The former case formed a mass lesion and was appropriately called a hibernoma. The latter case was interpreted to be nonneoplastic because it was limited to the marrow spaces and did not form a mass.

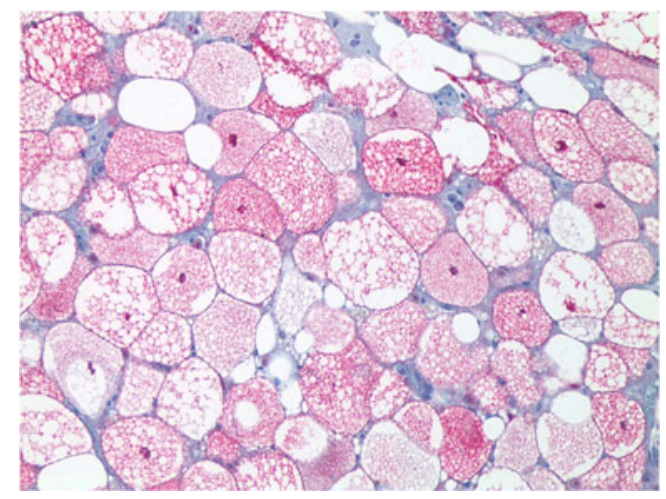

Fig. 4 Immunohistochemical stain for S100 using a red chromogen shows diffuse staining of multivacuolated cells $(\times 200)$ 
In our case, the fat cells have completely replaced a significant portion of the biopsy fragment and extend to the edge of the biopsy. This growth pattern suggests a neoplastic process. Therefore, the diagnosis of hibernoma is favored. Radiographic studies are not available to confirm the extent of the lesion.

Immunohistochemical staining for the purpose of distinguishing brown fat and hibernomas is very limited. S100 is well known to be positive in both. CD31 staining has been reported in three hibernomas as well as in 15 examples of nonneoplastic brown fat [8]. Two examples of hibernomas, both extraosseous, in a case series were shown to have positive $\mathrm{p} 53$ staining [9].

Although staining is limited, misdiagnosis of a hibernoma is unlikely due to its characteristic appearance. Potential mimics include liposarcomas, granular cell tumors, and foamy macrophages. The lipoblast, seen in liposarcomas, tends to be a smaller cell with fewer vacuoles. Atypia is rarely seen in hibernomas but will be seen in liposarcomas. Macrophages and granular cell tumors also stain with S100. However, granular cell tumors lack intracytoplasmic vacuoles. Macrophages will also stain for CD68.

The fact that two of these three cases are associated with ET and JAK2 mutations is curious. To evaluate for the presence of this mutation, the brown fat cells were dissected out of the paraffin-embedded tissue and analyzed for JAK2 V617F mutation by PCR analysis. No mutation was found. The test is reported to detect mutations when affected cells comprise at least $1 \%$ of the sample.
To date, all of the cases of intraosseous brown fat have occurred in females, at least in the sixth decade. Hibernomas and brown fat are typically of little to no clinical consequence. Knowledge of their existence in unusual locations is important to prevent misdiagnosis. This case describes a third example of this rare occurrence.

\section{References}

1. Lichtenbelt W, Vanhommerig J, Smulders N et al (2009) Coldactivated brown adipose tissue in healthy men. N Engl J Med 360 (15): $1500-1508$

2. Furlong M, Fanburg-Smith J, Miettinen M (2001) The morphologic spectrum of hibernoma. Am J Surg Pathol 25(6):809-814

3. Gaffney E, Hargreaves H, Semple E, Vellios F (1982) Hibernoma: distinctive light and electron microscopic features and relationship to brown adipose tissue. Hum Pathol 14(8):677-687

4. Mertens F, Rydholm A, Brosjo O, Willen H, Mitelman F, Mandahl N (1994) Hibernomas are characterized by rearrangements of chromosome bands 11q13-21. Int J Cancer 58:503-505

5. Mrozek K, Karakousis C, Bloomfield C (1994) Band 11q13 is nonrandomly rearranged in hibernomas. Gene Chromosome Cancer 9:145-147

6. Kumar R, Deaver M, Czerniak B, Madewell J (2011) Intraosseous hibernoma. Skeletal Radiol 40:641-645

7. Thorns C, Schardt C, Katenkamp D, Kahler C, Merz H, Feller A (2007) Hibernoma-like brown fat in the bone marrow: report of a unique case. Virchows Arch 452(3):343-345

8. Rosso R, Lucioni M (2006) Normal and neoplastic cells of brown adipose tissue express the adhesion molecule CD31. Arch Pathol Lab Med 130:480-482

9. Lele S, Chundru S, Chaljub G, Adegboyega P, Haque A (2002) Hibernoma: a report of 2 unusual cases with a review of the literature. Arch Pathol Lab Med 126:975-978 